\title{
X-Ray Versus Magnetic Resonance Imaging in Diabetic Foot Osteomyelitis: A Clinical Comparison
}

\author{
Gariani, Karim ; Lebowitz, Dan ; Kressmann, Benjamin ; Gariani, Joanna ; Uçkay, Ilker
}

\begin{abstract}
OBJECTIVE Radiographic imaging is an important diagnostic tool in diabetic foot osteomyelitis (DFO). It is unknown whether DFO cases diagnosed with conventional X-ray versus positive Magnetic Resonance Imaging (MRI) differ regarding epidemiology and treatment outcome. Theoretically, signs of inflammation on MRI without bone lesions might be easier to treat and predominate among selected clinical variables. METHODS Our clinical pathway for diabetic foot infections discourages the use of MRI for the diagnosis of DFO. We compared the epidemiology and therapy of non-amputated DFO with positive features on conventional X-ray, MRI, or both. Radiology specialists interpreted the images. The intraoperative aspect of bone during amputation and the results of bone cultures were considered gold standard for DFO diagnosis. RESULTS We prospectively followed 390 DFO episodes in 186 adult patients for a median of 2.9 years and performed 318 conventional X-rays (median costs 100 Swiss Francs; 100 US)and47(47/390;12\%)MRIscans(median800SwissFrancs; $800 U S)$. Among them, 18 episodes were associated with positive MRI findings but lacked bone lesions on X-ray. After debridement, the median duration of systemic antibiotics was 28 days for MRI-only episodes and 30 days for X-ray-positive cases (Wilcoxon-ranksum-test; $\mathrm{p}=0.26$ ). The corresponding median numbers of surgical debridements were 1 and 1 ; and remission was achieved in $25 \%$ and $27 \%$, respectively. In multivariate logistic regression analysis, MRI-only episodes did not alter remission rate (odds ratio 0.5, 95\% CI 0.1-5.2). CONCLUSIONS According to our clinical pathway, DFO episodes with positive MRI findings only did not differ epidemiologically and did not influence the choice of therapy nor remission rate.
\end{abstract}

DOI: https://doi.org/10.2174/1573399816999200729124134

Posted at the Zurich Open Repository and Archive, University of Zurich

ZORA URL: https://doi.org/10.5167/uzh-199328

Journal Article

Published Version

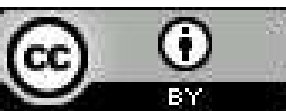

The following work is licensed under a Creative Commons: Attribution 4.0 International (CC BY 4.0) License.

Originally published at:

Gariani, Karim; Lebowitz, Dan; Kressmann, Benjamin; Gariani, Joanna; Uçkay, Ilker (2021). X-Ray Versus Magnetic Resonance Imaging in Diabetic Foot Osteomyelitis: A Clinical Comparison. Current Diabetes Reviews, 17(3):373-377.

DOI: https://doi.org/10.2174/1573399816999200729124134 
The below article has been published as a "full text ahead of publication" on the basis of the initial materials provided by the author. Once the paper is finalized and is ready to be published, this version will be removed. Full Text Ahead of Publication is a benefit provided to our authors to get their research published as soon as possible. The Editorial Department reserves the right to make modifications for further improvement of the manuscript in the final version.

\title{
Current Diabetes Reviews
}

\author{
Year 2020 \\ ISSN: $1874-6417$ (Online) \\ ISSN: 1573-3998 (Print)
}

\section{X-Ray Versus Magnetic Resonance Imaging in Diabetic Foot Osteomyelitis: A Clinical Comparison}

\author{
Karim Gariani ${ }^{1,2}$, Dan Lebowitz ${ }^{1,3}$, Benjamin Kressmann², Joanna Gariani ${ }^{4}$, Ilker Uçkay ${ }^{2,5}$
}

\begin{abstract}
${ }^{l}$ Division of Endocrinology, Diabetes, Hypertension and Nutrition, Geneva University Hospitals and Faculty of Medicine, Geneva, Switzerland; ${ }^{2}$ Clinical Pathway of Diabetic Foot Infections; ${ }^{3}$ Infection Control Unit \& Division of Infectious Diseases, University of Geneva Hospitals and Medical School, 1211 Geneva, Switzerland; ${ }^{4}$ Department of Radiology, Geneva University Hospitals, Geneva, Switzerland; 5 Department of Orthopedics, Balgrist University Hospital, University of Zürich, Zürich, Switzerland; Department of Infectiology, Balgrist University Hospital, University of Zürich, Zürich, Switzerland.
\end{abstract}

\begin{abstract}
Objective: Radiographic imaging is an important diagnostic tool in diabetic foot osteomyelitis (DFO). It is unknown whether DFO cases diagnosed with conventional X-ray versus positive Magnetic Resonance Imaging (MRI) differ regarding epidemiology and treatment outcome. Theoretically, signs of inflammation on MRI without bone lesions might be easier to treat and predominate among selected clinical variables.
\end{abstract}

\begin{abstract}
Methods: Our clinical pathway for diabetic foot infections discourages the use of MRI for the diagnosis of DFO. We compared the epidemiology and therapy of non-amputated DFO with positive features on conventional X-ray, MRI, or both. Radiology specialists interpreted the images. The intraoperative aspect of bone during amputation and the results of bone cultures were considered gold standard for DFO diagnosis.

Results: We prospectively followed 390 DFO episodes in 186 adult patients for a median of 2.9 years and performed 318 conventional X-rays (median costs 100 Swiss Francs; 100 US\$) and 47 (47/390; 12\%) MRI scans (median 800 Swiss Francs; 800US\$). Among them, 18 episodes were associated with positive MRI findings but lacked bone lesions on X-ray. After debridement, the median duration of systemic antibiotics was 28 days for MRI-only episodes and 30 days for X-raypositive cases (Wilcoxon-ranksum-test; $p=0.26$ ). The corresponding median numbers of surgical debridements were 1 and 1 ; and remission was achieved in $25 \%$ and $27 \%$, respectively. In multivariate logistic regression analysis, MRI-only episodes did not alter remission rate (odds ratio $0.5,95 \% \mathrm{CI} 0.1-5.2$ ).
\end{abstract}

Conclusions: According to our clinical pathway, DFO episodes with positive MRI findings only did not differ epidemiologically and did not influence the choice of therapy nor remission rate.

Keywords: Diabetic foot osteitis; X-ray; MRI; clinical associations; outcomes.

*Address correspondence to this author at the Balgrist University Hospital, Forchstrasse 340, 8008 Zürich / Switzerland; Tel: ++41 443863705 , Fax: ++41 4438637 09; E-mail: ilker.uckay@balgrist.ch 


\section{INTRODUCTION}

Recent studies failed to identify a minimal optimal duration of systemic antibiotic therapy for chronic osteomyelitis, especially in diabetic foot osteomyelitis (DFO)[1-8]. Since DFO might be clinically silent, radiologic studies remain important for diagnosis of $\mathrm{DFO}[9,10]$, with multiple available modalities. Conventional X-ray falls behind, with an $80 \%$ specificity and a $60 \%$ sensitivity due to significant delay in the occurrence of abnormal findings[9]. In contrast, Magnetic Resonance Imaging (MRI) detects inflammation in advance of established bone alterations, with a $90 \%$ sensitivity and $80 \%$ specificity $[9,11,12]$. DFO with mild signs of inflammation on MRI might be easier to treat than cases with more advance disease with alterations on conventional X-ray[13]. However, this assumption remains unverified. We compared the epidemiology and management of DFO with positive MRI findings only versus infections with both X-ray and MRI anomalies. This assessment may lead to a different approach for patients presenting DFO with only MRI anomalies such as a reduced antibiotherapy duration or reduced surgical approach.

\section{METHODS}

The Geneva University Hospitals ran a clinical pathway for adult DFO patients between 2013 and 2018. As part of a hospital-wide quality program, patients were not required to provide informed consent for storing key clinical data. Patients admitted to the orthopedic department of Geneva University hospital for an episode of DFO were included prospectively and analyzed retrospectively. Our clinical pathway discouraged using MRI for diagnosis of DFO, but it was performed in unclear cases. The diagnosis of DFO was based on radiological findings using X-ray and/or MRI. . Radiology specialists interpreted all MRIs and most X-rays. Both were performed within two weeks of admission. Patients with radiologically proven osteomyelitis were included in the analysis. Exclusion criteria were patients without both X-ray and MRI performed and patients requiring a immediate amputation for ischemia.

Radiologic signs used to define the presence of osteomyelitis on X-ray were lytic lesions, periosteal thickening, osteopenia, new bone apposition or loss of trabecular architecture. For MRI, the radiologic signs used to detect osteomyelitis were signal change from bone marrow edema (decreased on T1-weighted and increased on T2-weighted imaging sequences). We excluded episodes with less than three months of active follow-up, those with Charcot osteoarthropathy, and all DFOs with curative amputations. Those with partial amputations were included. We compared groups using the Pearson- $\chi^{2}$ or Wilcoxon-ranksum tests and performed a multivariate logistic regression analysis with the term "remission" as the outcome[14]. Analysis were performed using the STATA software (14.0, USA).

\section{RESULTS}

\section{General results and treatment}

Among 1071 adult diabetic foot infections, 390 DFOs met our study criteria (186 patients; 98 [25\%] females; median age, 69 years) (Figure 1). The median number of distinct DFO episodes per patient was two (range, 1-13 episodes). DFO mostly involved the forefoot (321 [83\%]). The mid- and hindfeet were involved in $44(11 \%)$ and $25(6 \%)$ cases, respectively. On admission, median transcutaneous oxygen tension on the forefoot was $30 \mathrm{mmHg}$ (interquartile range (IQR), $28-32 \mathrm{mmHg}$ ), median ankle-brachial index was 0.9 (IQR, 0.9-1.1), median serum C-reactive protein level was $76 \mathrm{mg} / \mathrm{L}$ (IQR, 64-87 mg/L), and most DFOs showed similar clinical signs of infection and necrosis. Thirty-five episodes were bacteremic and 248 occurred in patients with peripheral arterial disease. Debridement was performed in all cases, along with partial amputations in 294 (75\%). The median number of debridements was 1 (range, $0-7$ ). The median duration of post-surgical antibiotic therapy was 28 days, with a median parenteral administration of 6 days. We used 26 different antibiotic regimens on 53 distinct intraoperative combinations of microbiological results. 148 DFO episodes involved Staphylococcus aureus and 26 Pseudomonas aeruginosa infections. All patients were instructed in pressure offloading. Surgeons used vacuum-assisted negative pressure therapy in 24 episodes, and patients underwent hyperbaric oxygen therapy in 39 cases (median, 30 times). The duration between X-ray and first surgical procedure did not differ between both groups ( 7.5 vs 8.1 days; $p=0.51$ ).

\section{Radiological assessment}

We performed 381 conventional X-rays (381/390 episodes [98\%]; median costs, 100 Swiss Francs [100 US\$]) and 47 MRIs (47/390 [12\%]; median, 800 Francs [800 US\$]). Among the latter, only 38 scans were concomitantly accompanied by an X-ray. In 18 episodes, radiological anomalies were found on MRI only, and they were positive on both modalities in 20 episodes. In contrast, we found no single DFO case with established bone lesions on conventional X-ray without any signs on concomitant MRI.

\section{Outcomes and multivariate adjustment}

Overall clinical recurrence occurred after a median of 1 year following the previous episode. From a microbiological standpoint, concordance between the initial and subsequent DFOs was found in only $24 \%$ of cases (21/86 recurrences). We compared clinical features of DFOs with positive findings on MRI scan only, versus DFOs with other combinations of radiographic findings (Table 1). In this group comparison, MRI-only DFOs were not associated with particular variables, or with a lower rate of recurrence. In view of the considerable case-mix, we performed a multivariate logistic regression analysis (Table 2). The results confirmed the lack of association between MRI-only DFOs and remission rate. The goodness-of-fit result 


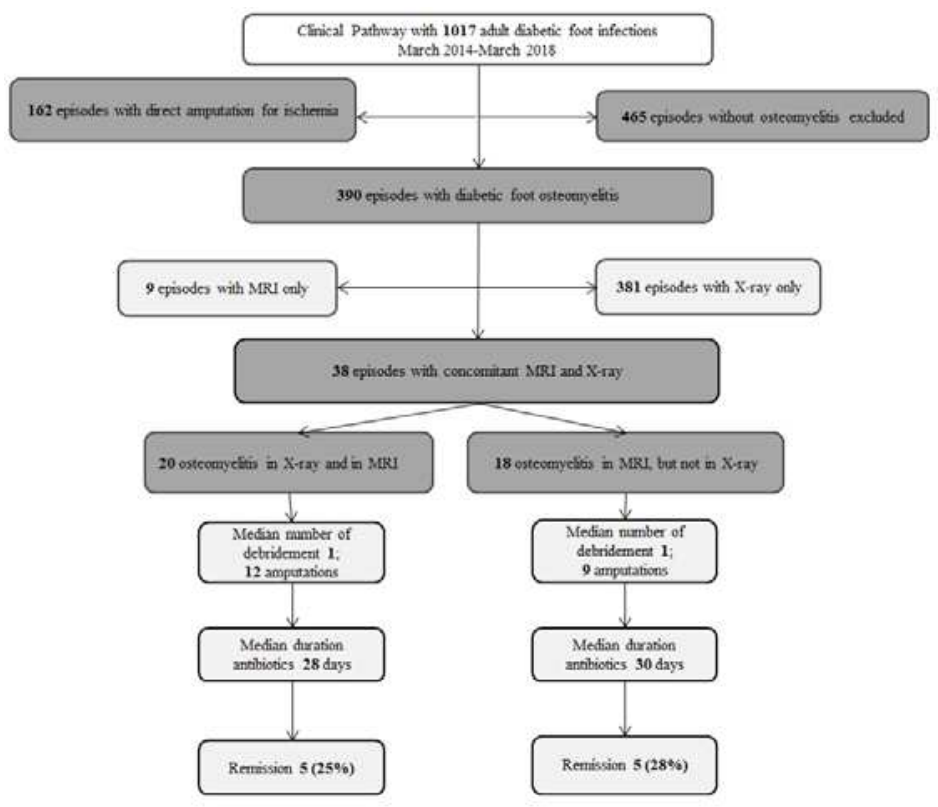

Fig. (1). Study flow chart.

of our final model was non-significant $(p=0.45)$ and the Receiver-Operating-Curve (ROC) value was 0.85 , highlighting a more than acceptable accuracy of our final model.

Table 1. Characteristics of diabetic foot osteomyelitis that was visible solely in MRI, but not in standard X-ray.

\begin{tabular}{|c|c|c|c|}
\hline $\mathbf{n}=\mathbf{3 8}$ & Visible in MRI \& X-ray, $n=20$ & $p$ value* & Visible only in MRI, $n=18$ \\
\hline Median BMI & $27.9 \mathrm{~kg} / \mathrm{m}^{2}$ & 0.84 & $28.1 \mathrm{~kg} / \mathrm{m}^{2}$ \\
\hline Glycosylated haemoglobulin & $7.2 \%$ & 0.74 & $7.4 \%$ \\
\hline Diabetic neuropathy & $16(80 \%)$ & 0.57 & $14(77.8 \%)$ \\
\hline Diabetic nephropathy & $9(45 \%)$ & 0.48 & $9(50 \%)$ \\
\hline Median serum C-reactive protein level & $59 \mathrm{mg} / \mathrm{L}$ & 0.81 & $71 \mathrm{mg} / \mathrm{L}$ \\
\hline White blood cell count & $11.86 \times 10^{3}$ & 0.64 & 12. $59 \times 10^{3}$ \\
\hline Calcaneal osteomyelitis & $1(5 \%)$ & 0.49 & $2(11 \%)$ \\
\hline $\begin{array}{c}\text { Mean duration between X-ray and first } \\
\text { surgical intervention }\end{array}$ & 7.5 days & 0.51 & 8.1 days \\
\hline Number of surgical interventions (median) & 1 (range, 0-6) & 0.83 & 1 (range, $0-5$ ) \\
\hline Partial amputation of bone & $12(60 \%)$ & 0.53 & $9(50 \%)$ \\
\hline Vacuum-assisted negative pressure therapy & $1(5 \%)$ & 0.45 & $2(12 \%)$ \\
\hline Duration of antibiotic treatment (median) & 28 days & 0.26 & 30 days \\
\hline Duration of intravenous antibiotics (median) & 10 days & 0.96 & 4 days \\
\hline
\end{tabular}

Significant $p$ values $\leq .05$ (two-tailed) are displayed in bold and italic. Pearson- $\chi^{2}$ and Wilcoxon-ranksum-tests, as appropriate 
Table 2. Logistic regression analyses with the outcome "remission" (odds ratio with 95\% confidence intervals)

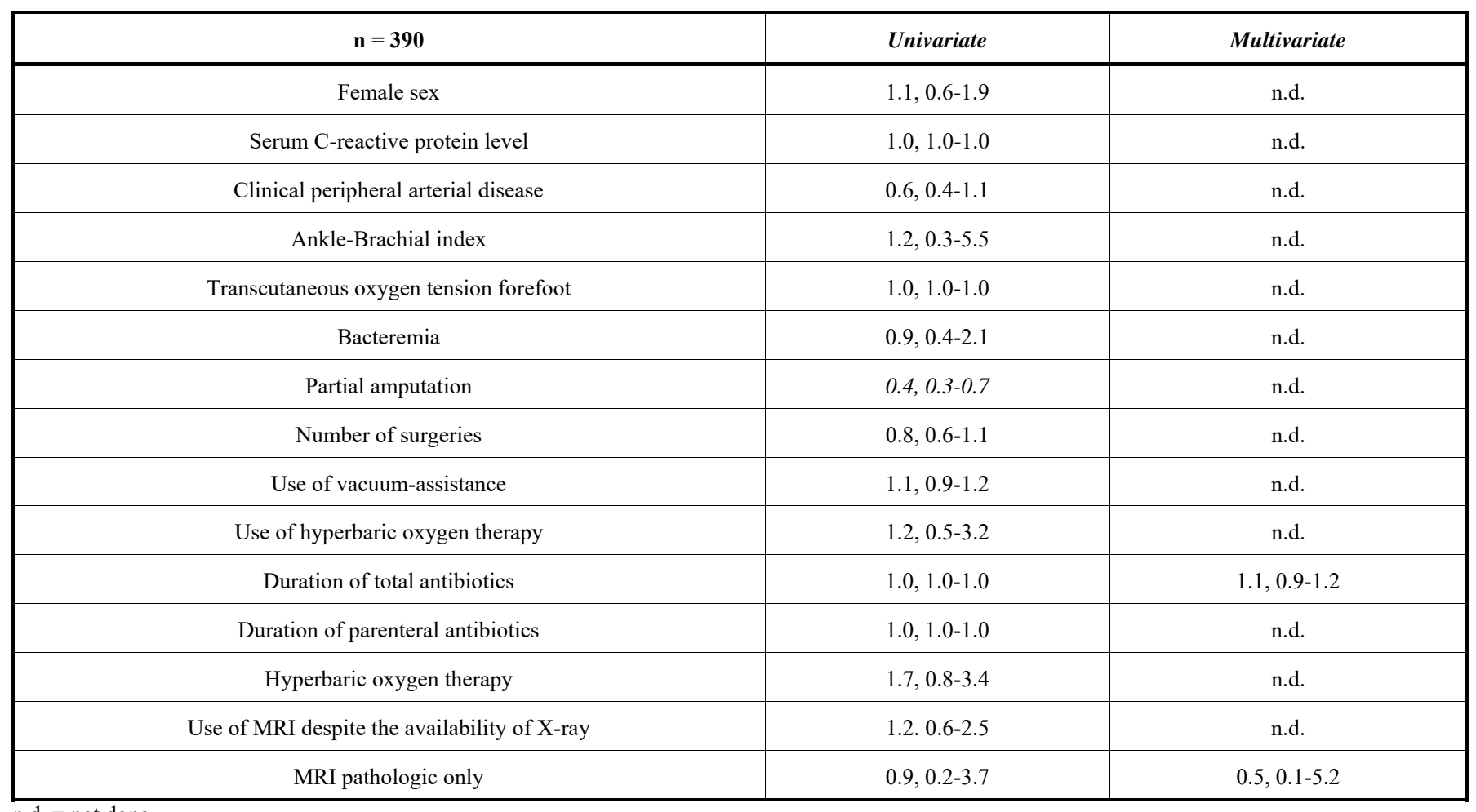

n.d. $=$ not done

\section{DISCUSSION}

In this single-center prospective clinical pathway of 390 adult DFO patients, episodes with abnormal findings on MRI only, but not on conventional X-ray, occurred very rarely (5\%). This subset of DFO cases was not associated with specific clinical parameters or differences in therapeutic outcomes. MRI positive-only DFO episodes were treated the same way as other DFO cases with X-ray lesions, and had a similar remission rate of $25 \%$ during the long-term follow-up. Moreover, neither total duration of antibiotic therapy nor the number of surgical debridements impacted the rate of remission, in accordance with the results our previous study. Only partial amputation was protective against clinical recurrence.

This study, apart from being retrospective, has several limitations. Firstly, patients who received treatment outside of our hospital may have been lost to follow-up. However, this is unlikely a major bias since our hospital has been the largest and only public reference center in the Geneva area for decades. Secondly, we focused mainly on moderate and severe DFO requiring hospitalization with potential surgery. Thus, our data may not reflect outcomes for mild cases that are treated entirely in the ambulatory setting. Thirdly, local wound care, especially pressure off-loading of the affected limb, is crucial for treating and preventing DFO. While the rationale of such measures is easily understandable, effectively implementing them depends on patient education and adherence, which we could not monitor. Fourthly, our definition of DFO, by design, relied on the positivity of bone culture. This definition is common, but might also lead to selection bias by excluding culture-negative cases, which might occur with prior antibiotic exposure[15]. However, our previous database studies of DFO patients failed to detect different treatment outcomes between culture-negative and culture-positive osteomyelitis, which speaks against a substantial bias. Finally, our hospital is set in a resource-rich country. Many centers worldwide either lack access to MRI, or tend to overuse it $[16,17]$. Our $12 \%$ prevalence regarding the use of MRI can be considered an intuitive balance between both.

\section{CONCLUSION}

In conclusion, according to our clinical pathway, DFOs with positive findings on MRI only did not differ in epidemiology or clinical outcomes after standardized treatment, when compared to those with findings on both MRI and conventional X-ray. Although MRI is a more sensitive modality, it does not seem to alter outcome by detecting early cases of DFO. This suggests that clinical management in terms of antibiotherapy or surgical procedure should not differ in patients with only positive MRI.

\section{ACKNOWLEDGMENTS}

We thank the Medical Directors of Geneva University Hospitals and the teams of the Bacteriology, Diabetology, Orthopaedics and Radiology for their support. 


\section{ETHICS APPROVAL AND CONSENT TO PARTICIPATE}

Not applicable.

\section{HUMAN AND ANIMAL RIGHTS}

No Animals/Humans were used for studies that are the basis of this research.

\section{CONSENT FOR PUBLICATION}

Not applicable.

\section{FINANCIAL FUNDING AND SPONSORSHIP}

Our hospital donated 50,000 CHF (Swiss Francs; equaling 50,000 US\$) for the Clinical Pathway of hospitalized DFI as a "quality of care improvement" project.

\section{DATA AVAILABILITY}

The data used to support the findings of this study are available from the corresponding author upon request.

\section{CONFLICT OF INTEREST}

KG, DL, SB and BK declare no conflict of interest. IU has received research donations from Innocoll Ltd. for another project.

All authors declare no financial support, grants, financial interests or consultancy that could lead to conflicts of interest.

\section{REFERENCES}

[1] Xing K, Huang G, Hua S, Xu G, Li M. Systematic review of randomized controlled trials on antibiotic treatment for osteomyelitis in diabetes. Diabet Med 2019; 36(5): 546-56. http://dx.doi.org/10.1111/dme.13935 PMID: 30785639

[2] Garcia Del Pozo E, Collazos J, Carton JA, Camporro D, Asensi V. Factors predictive of relapse in adult bacterial osteomyelitis of long bones. BMC Infect Dis 2018; 18(1): 635. http://dx.doi.org/10.1186/s12879-018-3550-6 PMID: 30526540

[3] Tone A, Nguyen S, Devemy F, et al. Six-week versus twelve-week antibiotic therapy for nonsurgically treated diabetic foot osteomyelitis: a multicenter open-label controlled randomized study. Diabetes Care 2015; 38(2): 302-7. http://dx.doi.org/10.2337/dc14-1514 PMID: 25414157

[4] Cortés-Penfield NW, Kulkarni PA. The History of Antibiotic Treatment of Osteomyelitis. Open Forum Infect Dis 2019; 6(5)ofz181 http://dx.doi.org/10.1093/ofid/ofz181 PMID: 31123692

[5] Pincher B, Fenton C, Jeyapalan R, Barlow G, Sharma HK. A systematic review of the single-stage treatment of chronic osteomyelitis. J Orthop Surg Res 2019; 14(1): 393. http://dx.doi.org/10.1186/s13018-019-1388-2 PMID: 31779664

[6] Gariani K, Lebowitz D, von Dach E, Kressmann B, Lipsky BA, Uçkay I. Remission in diabetic foot infections: Duration of antibiotic therapy and other possible associated factors. Diabetes Obes Metab 2019; 21(2): 244-51. http://dx.doi.org/10.1111/dom.13507 PMID: 30129109

[7] Lázaro Martínez JL, García Álvarez Y, Tardáguila-García A, García Morales E. Optimal management of diabetic foot osteomyelitis: challenges and solutions. Diabetes Metab Syndr Obes 2019; 12: 947-59. http://dx.doi.org/10.2147/DMSO.S181198 PMID: 31417295

[8] Masters EA, Trombetta RP, de Mesy Bentley KL, et al. Evolving concepts in bone infection: redefining "biofilm", "acute vs. chronic osteomyelitis", "the immune proteome" and "local antibiotic therapy". Bone Res 2019; 7: 20. http://dx.doi.org/10.1038/s41413-019-0061-z PMID: 31646012

[9] Glaudemans AW, Uçkay I, Lipsky BA. Challenges in diagnosing infection in the diabetic foot. Diabet Med 2015; 32(6): 748-59. http://dx.doi.org/10.1111/dme.12750 PMID: 25765225

[10] Giurato L, Meloni M, Izzo V, Uccioli L. Osteomyelitis in diabetic foot: A comprehensive overview. World J Diabetes 2017; 8(4): 135-42. http://dx.doi.org/10.4239/wjd.v8.i4.135 PMID: 28465790

[11] Lee YJ, Sadigh S, Mankad K, Kapse N, Rajeswaran G. The imaging of osteomyelitis. Quant Imaging Med Surg 2016; 6(2): 184-98. http://dx.doi.org/10.21037/qims.2016.04.01 PMID: 27190771

[12] Pineda C, Espinosa R, Pena A. Radiographic imaging in osteomyelitis: the role of plain radiography, computed tomography, ultrasonography, magnetic resonance imaging, and scintigraphy. Semin Plast Surg 2009; 23(2): 80-9. http://dx.doi.org/10.1055/s-0029-1214160 PMID: 20567730

[13] Toledano TR, Fatone EA, Weis A, Cotten A, Beltran J. MRI evaluation of bone marrow changes in the diabetic foot: a practical approach. Semin Musculoskelet Radiol 2011; 15(3): 257-68. http://dx.doi.org/10.1055/s-0031-1278425 PMID: 21644199

[14] Vittinghoff E, McCulloch CE. Relaxing the rule of ten events per variable in logistic and Cox regression. Am J Epidemiol 2007; 165(6): 710-8. http://dx.doi.org/10.1093/aje/kwk052 PMID: 17182981

[15] Rod-Fleury T, Dunkel N, Assal M, et al. Duration of post-surgical antibiotic therapy for adult chronic osteomyelitis: a single-centre experience. Int Orthop 2011; 35(11): 1725-31. http://dx.doi.org/10.1007/s00264-011-1221-y PMID: 21318568

[16] Mauler F, Wanivenhaus F, Böni T, Berli M. Nonsurgical Treatment of Osteomyelitis of the Hallux Sesamoids: A Case Series and Literature Review. J Foot Ankle Surg 2017; 56(3): 666-9. http://dx.doi.org/10.1053/j.jfas.2017.01.025 PMID: 28476396

[17] Roug IK, Pierre-Jerome C. MRI spectrum of bone changes in the diabetic foot. Eur J Radiol 2012; 81(7): 1625-9. http://dx.doi.org/10.1016/j.ejrad.2011.04.048 PMID: 21620598 